\title{
Epigenetic silencing of long non-coding RNA BM742401 in multiple myeloma: impact on prognosis and myeloma dissemination
}

\author{
Zhenhai Li ${ }^{1,2}$, Shaji Kumar ${ }^{3}$, Dong-Yan Jin ${ }^{4}$, George A. Calin ${ }^{5}$, Wee-Joo Chng ${ }^{6}$, Kam-Leung Siu ${ }^{4}$, Ming-Wai Poon ${ }^{2}$
} and Chor Sang Chim ${ }^{2 *}$

\begin{abstract}
Background: Long non-coding RNA (IncRNA) BM742401 is a tumor suppressor in gastric cancer and chronic lymphocytic leukemia. As the promoter and coding region of BM742401 are fully embedded in a CpG island, we hypothesized that BM742401 is a tumor suppressor IncRNA epigenetically silenced by promoter DNA methylation in multiple myeloma.
\end{abstract}

Methods: Methylation-specific PCR and quantitative bisulfite pyrosequencing were performed to detect the methylation of BM742401 in normal plasma cells, myeloma cell lines and primary myeloma samples. The expression of BM742401 was measured by qRT-PCR. The function of BM742401 in multiple myeloma cells was analyzed by lentivirus transduction followed by migration assay.

Results: BM742401 methylation was detected in 10 (66.7\%) myeloma cell lines but not normal plasma cells, and inversely correlated with expression of BM742401. In primary samples, BM742401 methylation was detected in 3 (12.5\%) monoclonal gammopathy of undetermined significance, 9 (15.8\%) myeloma at diagnosis and 8 (17.0\%) myeloma at relapse/progression. Moreover, BM742401 methylation at diagnosis was associated with inferior overall survival (median OS: 25 vs. 39 months; $P=0.0496$ ). In myeloma cell line JJN-3, stable overexpression of $B M 742401$ by lentivirus transduction resulted in reduced cell migration $(P=0.0001)$ but not impacting cell death or proliferation.

Conclusions: This is the first report of tumor-specific methylation-mediated silencing of BM742401 in myeloma, which is likely an early event in myelomagenesis with adverse impact on overall survival. Moreover, BM742401 is a tumor suppressor IncRNA by inhibiting myeloma cell migration, hence implicated in myeloma plasma cell homing, metastasis and disease progression.

Keywords: Multiple myeloma, BM742401, DNA methylation, Overall survival

\section{Background}

Multiple myeloma is one form of hematological malignancy characterized by the accumulation and patchy infiltration of the bone marrow by neoplastic plasma cells, which accounts for approximately $10 \%$ of all

\footnotetext{
*Correspondence: jcschim@hku.hk

${ }^{2}$ Department of Medicine, Queen Mary Hospital, The University of Hong Kong, Pokfulam Road, Pokfulam, Hong Kong, China

Full list of author information is available at the end of the article
}

hematologic malignancies [1]. Active multiple myeloma is characterized by $\geq 10 \%$ clonal plasma cells in the bone marrow in addition to the presence of endorgan damages, including hypercalcemia, renal failure, anemia, and/or lytic bone lesions, which are collectively known as CRAB features [2]. Multiple myeloma is often preceded by an entirely asymptomatic state, monoclonal gammopathy of undetermined significance (MGUS), that progresses into symptomatic myeloma at 
the rate of $1 \%$ per year [3]. Genetically, multiple myeloma is a heterogeneous disease with about half of the patients carrying non-hyperdiploid karyotypes (such as recurrent translocations involving immunoglobulin gene located at 14q32), whereas the other half carrying hyperdiploid karyotype (such as trisomies of odd number chromosomes) [4]. Despite major advances, multiple myeloma remains an incurable disease $[5,6]$.

Long non-coding RNA (lncRNA) is a novel class of RNA molecules of $>200$ nucleotides in length without protein-coding capacity [7, 8]. Functionally, lncRNAs may regulate gene expression at both transcriptional and post-transcriptional levels, and hence are involved in multiple biological processes including development, differentiation or carcinogenesis $[9,10]$. In particular, IncRNAs have been shown to be associated with the pathogenesis of multiple myeloma [11, 12]. For instance, lncRNA CRNDE (colorectal neoplasia differentially expressed) was found to be upregulated in primary myeloma samples and cell lines as compared with healthy controls, and associated with poor OS, and knockdown of CRNDE inhibited myeloma cell proliferation and colony formation and increased apoptosis and cell cycle arrest in G0/G1 phase [13], suggesting an oncogenic role for $C R N D E$ in myeloma. On the other hand, knockdown of lncRNA OIP5-AS1 has been shown to promote myeloma cell proliferation, cell cycle progression and inhibit apoptosis, suggesting OIP5-AS1 is a tumor suppressor in myeloma [14].

DNA methylation is an epigenetic mechanism for gene regulation without alteration of the DNA sequence [15], which refers to the addition of a methyl $\left(-\mathrm{CH}_{3}\right)$ group to carbon five position of the cytosine ring in a CpG dinucleotide catalyzed by DNA methyltransferases [16]. DNA regions enriched with CpG dinucleotides are called CpG islands $[17,18]$. In the mammalian genome, promoter-associated CpG islands are localized to or in close proximity to the promoter region of more than half of the human genes [19], and involved in the regulation of gene expression by DNA methylation [20]. Aberrant promoter DNA methylation contributes to carcinogenesis including blood cancers [21]. In normal cells, majority of promoter-associated $\mathrm{CpG}$ islands are unmethylated, associated with a euchromatin configuration, and hence transcriptionally ready or active for gene expression [22]. In contrast, cancer cells are characterized by global DNA hypomethylation, and locus-specific hypermethylation of promoter-associated CpG islands of tumor-suppressor genes, resulting in downregulation, and hence loss of tumor suppressor functions [23-25]. For instance, long non-coding RNA KIAA0495 has been shown to be silenced by promoter DNA methylation in myeloma [26].
By RNA-seq, $B M 742401$, localized to $18 \mathrm{q} 11.2$, was found to be downregulated in gastric cancer cells compared with normal tissues, which was associated with poor survival in patients with gastric cancer, and hence a potential tumor suppressor. Moreover, ectopic overexpression of BM742401 inhibited gastric cancer metastasis through regulation of cell migration and invasion [27]. Recently, in chronic lymphocytic leukemia (CLL), BM742401 was also found to be a tumor suppressor lncRNA, which was frequently methylated in primary samples of CLL [28]. As the promoter and coding region of $B M 742401$ are fully embedded in a CpG island, we hypothesized that BM742401 may also be a tumor suppressor lncRNA epigenetically silenced by promoter DNA methylation in multiple myeloma. To verify this hypothesis, we studied the methylation status of BM742401 promoter in healthy controls, myeloma cell lines and myeloma primary samples, and investigated its tumor suppressor function.

\section{Methods \\ Patient information}

Bone marrow samples were obtained from patients with MGUS $(\mathrm{n}=24)$, newly diagnosed myeloma $(n=57)$ and myeloma relapse/progression $(n=47)$. Diagnosis of myeloma was based on standard criteria of the International Myeloma Working Group (IMWG) [29]. Complete staging work-up consisted of bone marrow examination, skeletal imaging, serum and urine protein electrophoresis, and/or serum free light chain levels. Of the 57 patients with newly diagnosed myeloma, there were 24 females and 33 males, with a median age of 71 (35-88) years. Apart from 11 patients lacking International Staging System (ISS) data [30], there were 10 stage I, 22 stage II, and 14 stage III cases. There were 12 IgA, 40 IgG, 4 light chain, and 1 nonsecretary myelomas. According to the IMWG criteria, "relapse" was defined as the reappearance of the same paraprotein detected by serum/urine protein electrophoresis, appearance of new bone lesion or extramedullary plasmacytoma, or unexplained hypercalcemia after prior complete remission; while "progression" as increase of M-protein by $25 \%$ from lowest confirmed response value with an absolute rise of serum M-protein of $\geq 0.5 \mathrm{~g} / \mathrm{dL}$ [31]. The study has been approved by the Institutional Review Board of Queen Mary Hospital (UW 05-269 T/932), and written informed consent was obtained from patient for publication of this article and any accompanying data or images. DNA of patient samples are extracted from bone marrow buffy coat, whereby malignant plasma cells are enriched by ficoll gradient centrifugation. 


\section{Cell culture}

Human myeloma cell lines (HMCLs) KMS-12-PE, MOLP-8, OPM-2 and U-266 were obtained from Deutsche Sammlung von Mikroorganismen und Zellkulturen (DSMZ, Braunschweig, Germany). NCI-H929 was purchased from American Type Culture Collection (ATCC, Manassas, VA, USA). KMS-11/BTZ and OPM-2/ BTZ were acquired from Kyowa Hakko Kirin Co. Ltd. (Tokyo, Japan). LP-1 and RPMI-8226 were kindly provided by Prof. Robert Orlowski (Department of Lymphoma/Myeloma, Division of Cancer Medicine, The University of Texas MD Anderson Cancer Center, Houston, TX, USA). JJN-3, OCI-MY5 and RPMI-8226R were kindly provided by Prof. Wee Joo Chng (Department of Medicine, Yong Loo Lin School of Medicine, National University of Singapore). WL-2 was kindly provided by Prof. Andrew Zannettino (Myeloma Research Programme, The University of Adelaide, Australia). MMLAL [32] and MMKKF (unpublished) were established from the myelomatous pleural effusion of myeloma patients. Cell lines were cultured in RPMI-1640 medium (IMDM for LP-1, DMEM + IMDM for MMLAL), supplemented with $10 \%$ or $20 \%$ fetal bovine serum, $50 \mathrm{U} / \mathrm{mL}$ of penicillin and $50 \mu \mathrm{g} / \mathrm{mL}$ streptomycin, in a humidified atmosphere of $5 \% \mathrm{CO}_{2}$ at $37{ }^{\circ} \mathrm{C}$. All culture reagents were purchased from Invitrogen (Carlsbad, CA, USA).

\section{Methylation-specific polymerase chain reaction (MSP)}

Detailed procedures of MSP have been previously described [33, 34]. Primer sequences and conditions are in Table 1.

\section{Quantitative bisulfite pyrosequencing}

With bisulfite-treated DNA of HMCLs as template, specific PCR product overlapping the MSP amplicon was amplified by a pair of methylation-unbiased primers using PyroMark PCR Kit (Qiagen). Primer sequences are as followed: forward primer: $5^{\prime}$-AGG GGA GGA GAG AAA AGA G-3'; reverse primer: 5'-biotin-AAC TAT ACA CTA CCA ACT CCT-3'; condition: $2 \mathrm{mM}$ $\mathrm{MgCl}_{2} / 61{ }^{\circ} \mathrm{C} / 50$ cycles. PCR product was purified and consecutive $\mathrm{CpG}$ dinucleotides was pyrosequenced with sequencing primer: 5'-GTT TAG GTA GAT AAT GAG AGT-3' [28].

\section{Quantitative reverse transcription polymerase chain reaction (qRT-PCR)}

Total RNA was isolated using mirVana ${ }^{\mathrm{TM}}$ miRNA Isolation Kit (Ambion, Austin, TX, USA). Reverse transcription was performed using QuantiTect Reverse Transcription Kit (Qiagen). BM742401 was quantified using SYBR Green Master Mix (Applied Biosystems, Waltham, MA, USA) with GAPDH as endogenous control. Primer sequences of qRT-PCR for BM742401 and GAPDH were listed in Table 1. Expression of BM742401 was calculated by $\triangle C T$ method.

\section{5-Aza-2'-deoxycytidine (5-AzadC) treatment}

MOLP-8 cells, which were completely methylated for $B M 742401$, were treated with $0.5 \mu \mathrm{mol} / \mathrm{l}, 1 \mu \mathrm{mol} / \mathrm{l}$ and $1.5 \mu \mathrm{mol} / 1$ 5-AzadC (Sigma-Aldrich, St. Louis, MO, USA) in fresh medium replaced every $24 \mathrm{~h}$ for 5 days. Cells were harvested for DNA and RNA extraction on day 5. Relative expression level of BM742401 in 5-AzadCtreated group against untreated group was calculated by $2^{-\Delta \Delta \mathrm{CT}}$ method.

\section{Lentivirus transduction}

The full-length cDNA of BM742401 was amplified and cloned into the XbaI and EcoRI sites of a PCDHCMVMCS-EF1-copGFP lentivector (System Biosciences, Palo Alto, CA, USA; named empty vector) as described before [28], and the reconstructed vector was named $B M 742401$ vector. $B M 742401$ vector and empty vector were then co-transfected with pPACK packaging plasmid mix respectively into $293 \mathrm{TN}$ cells, followed by collection of supernatants at $48 \mathrm{~h}$ after transfection and concentration of pseudoviral particles by PEG-it ${ }^{\mathrm{TM}}$ Virus Precipitation Solution (System Biosciences). After pseudoviral titer estimation using $293 \mathrm{TN}$ cells, JJN-3 cells were transduced for $48 \mathrm{~h}$ by the pseudoviral particles with multiplicity of infection at 4 . GFP-positive JJN-3 cells were

Table 1 Primer sequences and PCR reaction conditions for BM742401

\begin{tabular}{|c|c|c|c|c|}
\hline Primer set & Forward primer $\left(5^{\prime}-3^{\prime}\right)$ & Reverse primer $\left(5^{\prime}-3^{\prime}\right)$ & $\mathrm{MgCl}_{2} / \mathrm{Tm} /$ cycles & References \\
\hline \multicolumn{5}{|c|}{ (I) BM742401 MSP } \\
\hline M-MSP & CGTTTA GGT AGA TAA TGA GAG TCG C & AAA TCA AAC GTT CTA TAA CCT CCG & $1.5 \mathrm{mM} / 60^{\circ} \mathrm{C} / 38 \mathrm{X}$ & [28] \\
\hline U-MSP & TGT GTT GTT TAG GTA GAT AAT GAG AGT TGT & CCA AAT CAA ACA TTC TAT AAC CTC CA & $2.0 \mathrm{mM} / 60^{\circ} \mathrm{C} / 38 \mathrm{X}$ & \\
\hline \multicolumn{5}{|l|}{ (II) qRT-PCR } \\
\hline BM742401 & TTG GTT CTT TTC TAC AAG GAT GTC & CGA ATC GGT CAA TGT CCA CC & NA & NA \\
\hline GAPDH & ACC ACA GTC CAT GCC ATC ACT & TCC ACC ACC CTG TTG CTG TA & NA & NA \\
\hline
\end{tabular}

Tm, annealing temperature; M-MSP, methylated MSP; U-MSP, unmethylated MSP 
selected by flow cytometry (BD FACSAria I Cell Sorter) and further cultured for 3 weeks. Relative expression of $B M 742401$ in response to transduction of BM742401 vectors as compared with empty vectors was analyzed by $2^{-\Delta \Delta \mathrm{CT}}$ method.

\section{Migration assay}

To test the effect of BM742401 overexpression on myeloma cell migration, we used bone marrow stromal cells (BMSCs) as a source for secreting chemoattractant for myeloma cells.

In the migration assay, a pilot transwell experiment was conducted to find out the optimal experimental conditions. At $24 \mathrm{~h}$ before migration assay, JJN-3 cells transduced with empty vector were starved by washing with PBS and resuspending in RPMI-1640 medium without FBS. The next day, in each of the transwell permeable support $(8.0-\mu \mathrm{m}$ polycarbonate membrane, $6.5-\mathrm{mm}$ insert, and 24-well plate; Corning Costar, Tewksbury, MA, USA), $1 \times 10^{5}$ starved JJN-3 cells were seeded in $200 \mu \mathrm{l} \mathrm{RPMI}-1640$ medium. In the lower chamber, one of the following three conditions was used: (1) $500 \mu \mathrm{l}$ RPMI-1640 medium with 20\% FBS; (2) $500 \mu \mathrm{l}$ BMSC conditioned medium (described below); or (3) $2 \times 10^{5}$ BMSCs in $500 \mu \mathrm{l}$ DMEM medium with $10 \%$ FBS that had been seeded on the day before. BMSCs were cultured from normal bone marrow donors as previously described [35]. "BMSC conditioned medium" was generated by mixing the filtered culture medium of BMSCs (at $37{ }^{\circ} \mathrm{C}$ in $5 \% \mathrm{CO}_{2}$ for $24 \mathrm{~h}$ ) with $20 \%$ FBS in RPMI1640 medium at the ratio of $1: 1$. After $72 \mathrm{~h}$ of incubation at $37^{\circ} \mathrm{C}$, the GFP-positive cells that had migrated to the lower chambers were counted using fluorescence microscope (Axiovert 135, ZEISS microscopy, Germany). The rationale of the use of BMSCs in the lower chamber stemmed from the concept that myeloma cell homing is mediated by SDF-1 produced by BMSCs that bind to the CXCR4 receptor on myeloma cells, hence myeloma cells would migrate to the bone marrow niches due to this concentration gradient [36]. As the highest myeloma cell migration occurred with BMSCs laid at the bottom of the lower chamber (Additional file 1: Figure S1), which hence was adopted for subsequent transwell experiments to compare migration efficiency between JJN-3 cells transduced with empty vector and $B M 742401$ vector. Triplicate experiments were performed for each group, and the means and standard deviations were calculated.

\section{Trypan blue exclusion assay}

Cell death was analyzed by trypan blue (Sigma-Aldrich) at day 3 after seeding cells. Cells in five random microscopic fields were counted for each group under microscope. Dead cell (stained in blue) percentage $=$ average number of dead cells per microscopic field/average number of total cells per microscopic field.

\section{MTS assay}

The number of viable cells in proliferation was measure by CellTiter $96^{\circledR} \mathrm{AQ}_{\text {ueous }}$ One Solution Cell Proliferation Assay Kit (Promega, Madison, WI, USA) following the manufacturers' instructions. Relative proliferation percentage of BM742401 overexpressed cells compared with control cells was calculated at day 5 after seeding cells.

\section{Statistical analysis}

Overall survival (OS) was measured from the date of diagnosis to the date of last follow-up or death. OS of patients with and without BM742401 methylation were compared. Survival was plotted by the Kaplan-Meier method, and compared by the log-rank test. The difference between JJN-3 cells transduced with BM742401 vectors and empty vectors in migration assay was studied by Student's $t$ test. All $P$ values were two-sided and $P<0.05$ was defined as significant difference.

\section{Results \\ Methylation of $B M 742401$ in healthy controls and human myeloma cell lines (HMCLs)}

MSP was carried out to examine methylation of $B M 742401$ in the bisulfite-converted DNA of healthy controls [peripheral blood $(\mathrm{n}=10)$ and CD138-sorted bone marrow plasma cell $(n=7)]$ and HMCLs $(n=15)$. Direct sequencing of the M-MSP products from positive control with methylated DNA confirmed complete bisulfite conversion and MSP specificity, as indicated by conversion of all unmethylated cytosines into thymidines after PCR, whereas all methylated cytosines remained unchanged (Fig. 1a). None of the healthy controls showed methylation of BM742401 (Fig. 1b). By contrast, in HMCLs, BM742401 was completely methylated (MM; M-MSP positive but U-MSP negative) in KMS-12-PE, MOLP-8 and OCI-MY5, partially methylated (MU; both M-MSP and U-MSP positive) in JJN-3, LP-1, OPM-2, U-266, WL-2, OPM-2/BTZ and RPMI8226R, and completely unmethylated (UU; M-MSP negative but U-MSP positive) in NCI-H929, RPMI8226, MMKKF, MMLAL and KMS-11/BTZ (Fig. 1C). Moreover, these MSP methylation statuses (MM, MU, and UU) were verified using quantitative bisulfite pyrosequencing, which showed that completely methylated HMCLs were associated with a higher methylation level between $63.4 \%$ to $85.4 \%$, partially methylated HMCLs carried an intermediate methylation level of $36.9 \%$ to $49.6 \%$, and completely unmethylated HMCLs were associated with a lower methylation level from 
a Wild-type: $\quad$ CGAGTCCACCCGGGGGGCACCGAGCCCAGGGACCCCGGGGGAGGGGCG

Methylated: CGAGTTTATTCGGGGGGTATCGAGTTTAGGGATTTCGGGGGAGGGGCG
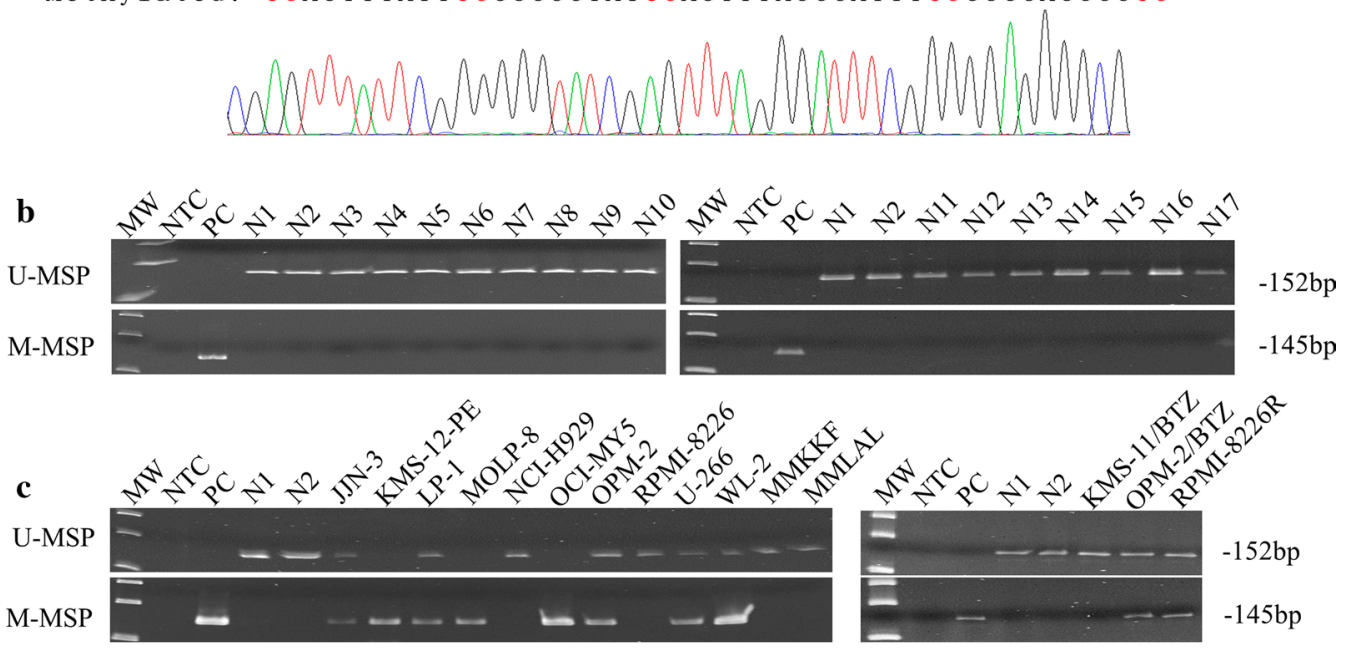

MW: marker; NTC: no template control; PC: positive control with methylated DNA;

N1-N10: normal peripheral blood control; N11-N17: normal bone marrow plasma cell control

Fig. 1 Methylation of BM742401 in healthy controls and HMCLs. a Direct sequencing of M-MSP products from positive control with methylated DNA showed the conversion of all unmethylated cytosines into uracils (turned into thymidines after PCR) but all methylated cytosines remained unchanged, indicating complete bisulfite conversion and specificity of MSP. b M-MSP and U-MSP showed that all healthy controls (N1-N17) were completely unmethylated (UU), whereas positive control with methylated DNA was completely methylated (MM). c M-MSP and U-MSP showed BM742401 was MM in HMCLs, including KMS-12-PE, MOLP-8 and OCI-MY5, partially methylated (MU) in JJN-3, LP-1, OPM-2, U-266, WL-2, OPM-2/BTZ and RPMI-8226R, UU in NCI-H929, RPMI-8226, MMKKF, MMLAL and KMS-11/BTZ

$15.1 \%$ to $23.6 \%$ (Additional file 2: Figure S2). These data suggested that BM742401 was methylated in a tumorspecific manner in myeloma.

\section{Methylation and expression of BM742401 in HMCLs}

To study if methylation was correlated with repression of $B M 742401$, qRT-PCR was employed to measure the expression levels of BM742401 in HMCLs. Results showed that HMCLs with methylation of $B M 742401$ had significantly lower expression levels of $B M 742401$ (Fig. 2a; MM vs. UU, $P=0.041 ; M M+M U$ vs. UU, $P=0.047)$ than HMCLs that were completely unmethylated.

To further testify if promoter DNA methylation resulted in downregulation of BM742401, MOLP-8 cells, which were completely methylated for $B M 742401$, were treated with 5-AzadC, a demethylation agent. Upon treatment with 5-AzadC, the promoter of BM742401 was demethylated as evidenced by the emergence of U-MSP signal on day 5 (Fig. 2b). Moreover, by qRT-PCR, BM742401 was simultaneously re-expressed by 5.2 to 9.9 folds with different concentrations of 5-AzadC (Fig. 2b). Therefore, in myeloma cells, methylation-mediated silencing of BM742401 was reversible.

\section{Methylation of BM742401 in primary bone marrow samples}

By MSP, methylation of BM742401 was detected in primary bone marrow samples of $3(12.5 \%)$ MGUS, 9 (15.8\%) myeloma at diagnosis, and 8 (17.0\%) myeloma at relapse/progression (Fig. 3a). Methylation frequency of BM742401 was not significantly different among those consecutive clinical stages of myeloma (MGUS vs. myeloma at diagnosis: $P=1.000$; myeloma at diagnosis vs. myeloma at relapse/progression: $P=1.000$ ). In contrast to absence of methylation in normal, presence of methylation in MGUS with a frequency comparable to consecutive stages from MGUS to myeloma at diagnosis and relapse/progression indicated BM742401 methylation might be an early event in the pathogenesis of myeloma.

Interestingly, by Kaplan-Meier analysis, the projected overall survival (OS) of diagnostic myeloma patients with and without BM742401 methylation were $11.1 \%$ and $45.8 \%$ respectively, and patients with BM742401 methylation $(n=9)$ showed significantly shorter OS than patients without BM742401 methylation $(\mathrm{n}=48$; Fig. 3b; median OS: 25 vs. 39 months; $P=0.0496$ ). 
a

BM742401 expression in HMCLs

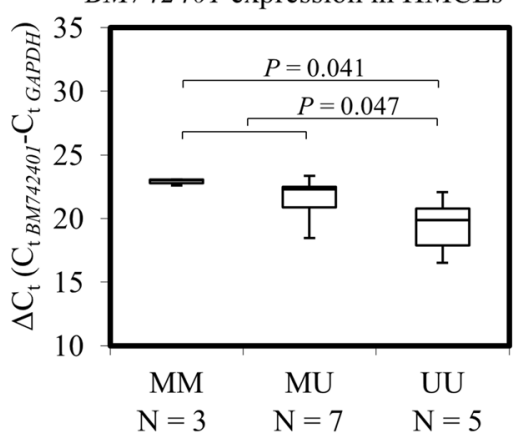

b
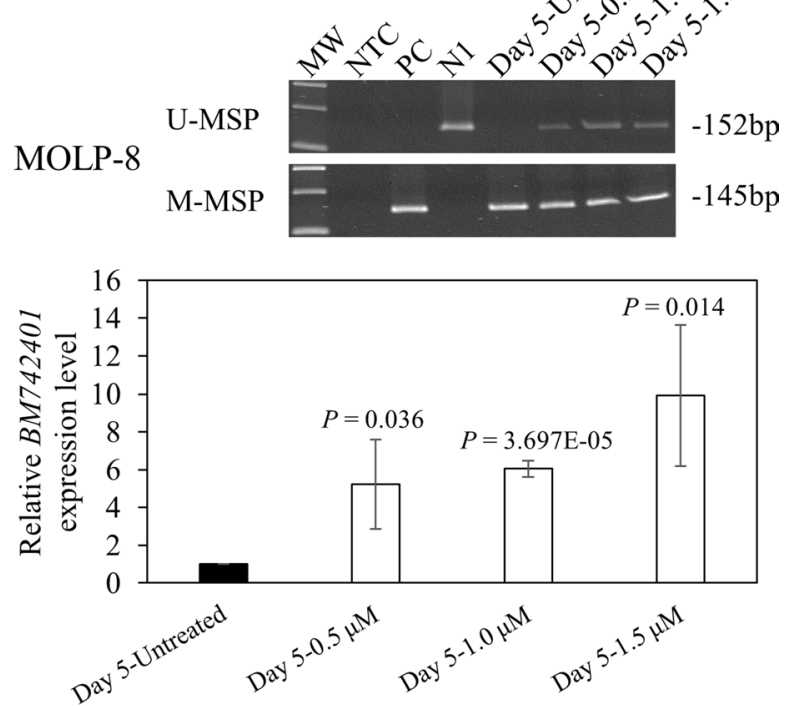

MW: marker; NTC: no template control; PC: positive control with methylated DNA; N1: normal peripheral blood control

Fig. 2 Methylation and expression of BM742401 in HMCLs. a By qRT-PCR, methylation of BM742401 was significantly correlated with lower expression level and hence larger $\triangle C$ t of $B M 742401$ (MM vs. UU, $P=0.041$; MM+ MU vs. UU, $P=0.047$ ). b In MOLP-8 cells, which were completely methylated for BM742401, treatment with 5-AzadC for 5 days led to BM742401 promoter demethylation, as evidenced by emergence of U-MSP signal (upper), and concomitant re-expression of BM742401 (lower). Error bars represent standard deviation from three independent qRT-PCR

\section{$\mathbf{a}$}
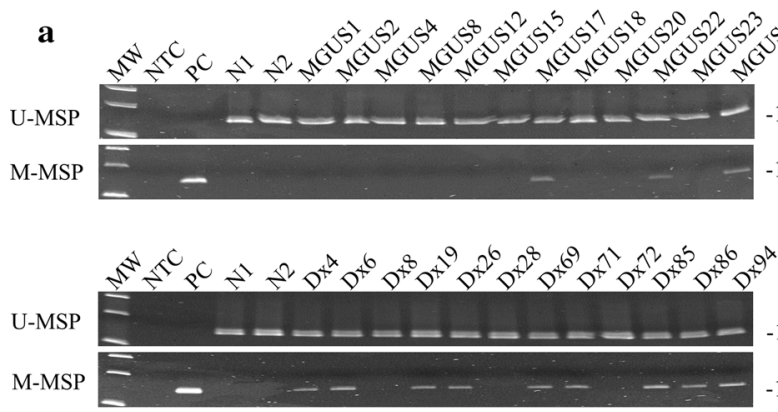

$-152 \mathrm{bp}$

$-145 \mathrm{bp}$

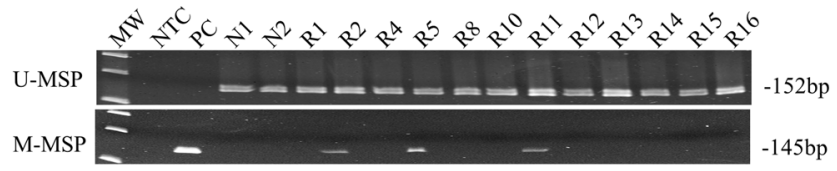

b

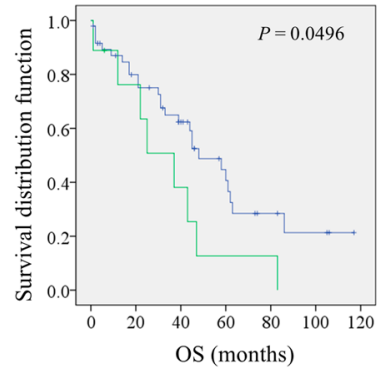

- BM742401 methylated diagnostic patients

- BM742401 unmethylated diagnostic patients

MW: marker; NTC: no template control; PC: positive control with methylated DNA;

N1, N2: normal control; MGUS: primary samples at MGUS; Dx: primary samples at

diagnosis; R: primary samples at relapse; OS: overall survival

Fig. 3 Methylation and expression of BM742401 in primary bone marrow samples. a Representative M-MSP and U-MSP showing methylation of BM742401 in primary samples of MGUS (total: $n=24$ ), myeloma at diagnosis (total: $n=57$ ) and myeloma at relapse/progression (total: $n=47)$. The numbers were assigned for illustration purpose, and hence, the identical Arabic numerals in different disease stages are not serial samples from the same patient. b Kaplan-Meier analysis of OS in patients with and without methylation of BM742401 
Tumor suppressive function of $B M 742401$ in myeloma cells As BM742401 was frequently methylated in HMCLs and primary samples, we postulated that it might act as a tumor suppressor. By lentivirus transduction, BM742401 was stably overexpressed by 9397.0 folds in JJN-3 cells compared with empty vector control (Fig. $4 \mathrm{a}$ and Additional file 3: Figure S3; $P=0.0009)$. Moreover, overexpression of BM742401 resulted in reduced cell migration of JJN-3 cells by transwell migration assay (Fig. $4 \mathrm{~b}$ and c; $P=0.0001$ ), but not affecting cell death (Fig. $4 \mathrm{~d}$; $P=0.1009$ ) or proliferation (Fig. 4e; $P=0.2401$ ) by trypan blue exclusion assay and MTS assay respectively. Therefore, BM742401 exhibits its tumor suppressor property in myeloma by inhibiting cell migration.

\section{Discussion}

\section{There are a number of interesting observations in this} study

Firstly, methylation of BM742401 was tumor-specific as it was absent in normal controls, whereas frequently detected in HMCLs and primary myeloma samples, which is similar to the tumor-specific methylation of other tumor suppressive protein coding genes [37, 38], miRNAs [39, 40] and lncRNA [26] in myeloma. In contrast, methylation of some miRNAs, such as miR9-2 and miR-373 [41, 42], occurred in both cancer cells and their normal counterparts, and hence methylated in a tissue-specific manner, thereby unimportant in carcinogenesis.

Secondly, methylation-mediated silencing of BM742401 was shown to be reversed by treatment of demethylating agent, consistent with the reversible silencing of BM742401 shown in CLL [28], indicating that promoter DNA methylation is also a mechanism for repression of tumor suppressor lncRNAs in myeloma.

Thirdly, in primary samples, methylation of BM742401 appeared as early as MGUS, at a frequency comparable to that of active myeloma at diagnosis and relapse/progression. Therefore, it is likely that methylation of BM742401 is an early event in the pathogenesis of myeloma, similar to methylation of $m i R-203$ [40] and $m i R-342$ [34]. By contrast, $m i R-129-2$ methylation was implicated in the progression from MGUS to symptomatic myeloma [43], and $m i R-34 b / c$ methylation at relapse/progression of myeloma [39]. Moreover, methylation of $B M 742401$ correlated with shorter OS in newly diagnosed myeloma, similar to CDKN2A [44, 45] and DAPK1 [46] methylation, suggesting an

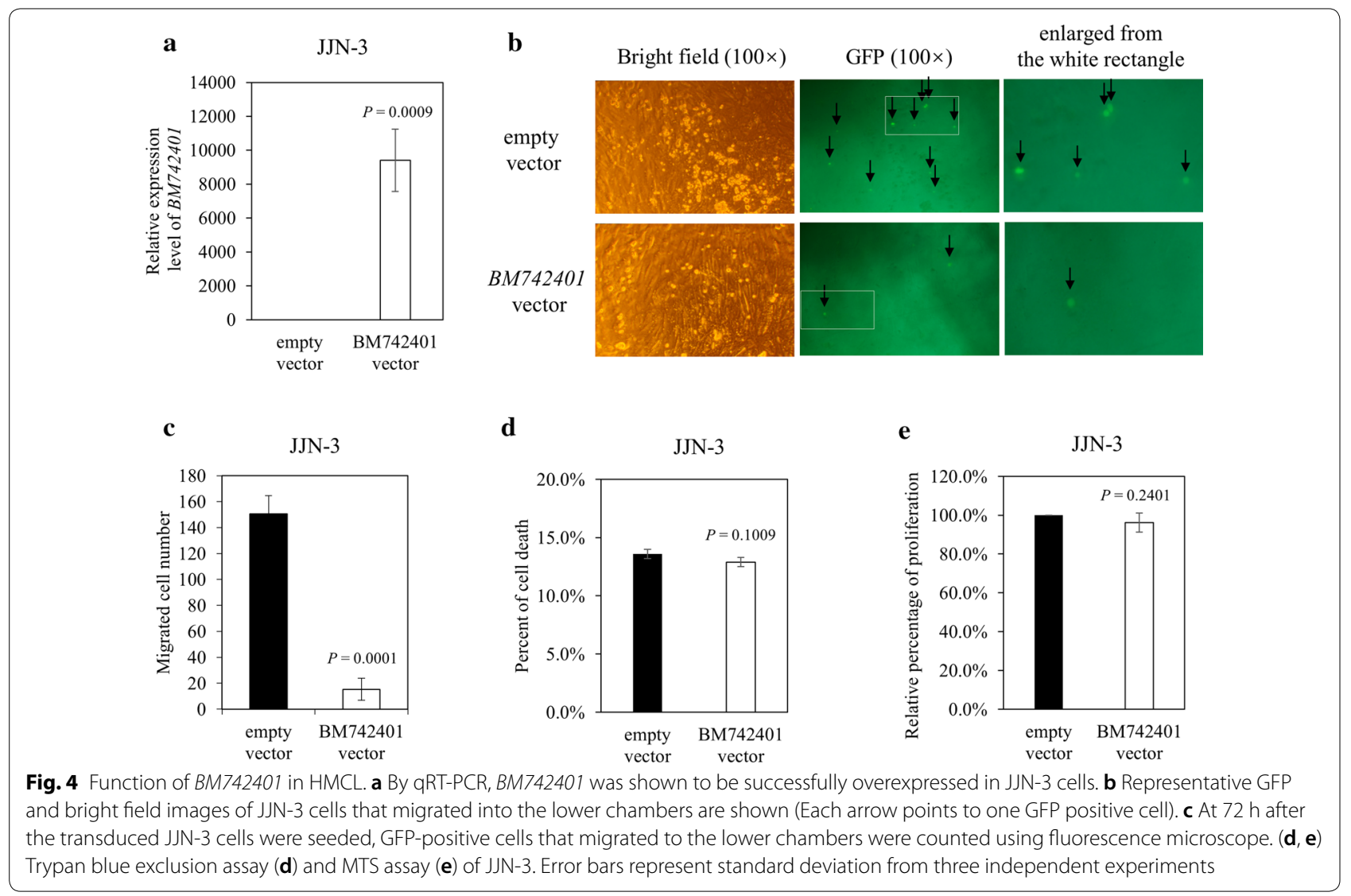


adverse impact of BM742401 methylation for OS. As this cohort of myeloma patients is small and not uniformly treated, the prognostic significance of BM742401 methylation needs to be verified by multivariate analysis in a larger cohort of uniformly-treated patients.

Fourthly, the tumor suppressor function of $B M 742401$ has been shown in gastric cancer [27] and CLL [28]. Herein, we confirmed BM742401 as a tumor suppressor in myeloma, as evidenced by the inhibition of myeloma cell migration in myeloma cells with stable overexpression of BM742401. In myelomagenesis, there is constant trafficking of myeloma cells through the blood to the bone marrow niches, a process termed homing, due to secretion of SDF-1 by BMSC, thereby creating a concentration gradient from the $\mathrm{BM}$ stroma to the circulating plasma cells [47]. Indeed, in our pilot migration experiment using one of the following three conditions in the lower chamber including FBS only, BMSC conditioned medium, or BMSCs, lower chamber loaded with BMSCs resulted in the highest yield of myeloma cell migration across the membrane (Additional file 1: Figure S1), implicating the importance of the SDF-1/CXCR4 axis in plasma cell migration [36]. Indeed, myeloma cells with stable overexpression of BM742401 led to significant inhibition of myeloma plasma cell migration than cells with empty vector using this condition. Therefore, epigenetic silencing of BM742401 may enhance myeloma metastasis and disease progression. This is consistent with the adverse impact of BM742401 methylation on OS in our cohort, hence warrants further investigation.

By contrast, overexpression of BM742401 did not influence myeloma cell death or proliferation, hence similar to the findings in gastric cancer that BM742401 inhibited gastric cancer cell migration and invasion but not cell viability [27], but different from CLL in that BM742401 inhibited CLL cell proliferation and enhanced apoptosis [28]. Therefore, the tumor suppressor function of BM742401 appears cancer-type specific.

Lastly, BM742401 localizes in an antisense direction to a neighboring protein-coding gene GATA6. As lncRNA may involve in the regulation of its neighboring gene, methylation of BM742401 and expression of GATA6 may be studied in myeloma. For example, HOTTIP expression was correlated to the activation of HOX genes, including $H O X A 7,9,10,11$, and 13 , in the HOXA locus [9]. Moreover, GATA6 may regulate the WNT signaling pathway, which is dysregulated in myeloma [48, 49], thereby methylation of BM742401 may link to the regulation of the Wnt signaling pathway, playing a role in the pathogenesis of myeloma.

\section{Conclusions}

In myeloma, methylation-mediated silencing of BM742401 is tumor-specific, reversible, associated with inferior OS, and likely an early event in myelomagenesis, and BM742401 is a tumor suppressor lncRNA by inhibiting myeloma cell migration, hence implicated in myeloma plasma cell homing and metastasis.

\section{Supplementary information}

Supplementary information accompanies this paper at https://doi. org/10.1186/s12935-020-01504-4.

Additional file 1: Figure S1. Pilot transwell migration assay. JJN-3 cells transduced with empty vector were starved and seeded on the transwell support.

Additional file 2: Figure S2.Quantitative bisulfite pyrosequencing analysis of BM742401 in HMCLs.

Additional file 3: Figure S3.Representative GFP (upper) and bright field images (lower) of JJN-3 cells stably transduced with empty vector or BM742401 vector.

\section{Abbreviations}

IncRNA: Long non-coding RNA; MGUS: Monoclonal gammopathy of undetermined significance; miRNA: microRNA; MSP: Methylation-specific PCR; HMCLs: Human myeloma cell lines; M-MSP: Methylated MSP; U-MSP: Unmethylated MSP; MM: Completely methylated; MU: Partially methylated; UU: Completely unmethylated; PCR: Polymerase chain reaction; qRT-PCR: Quantitative realtime PCR; 5-AzadC: 5-Aza-2'-deoxycytidine; OS: Overall survival.

\section{Acknowledgements}

Not applicable.

\section{Authors' contributions}

ZL and MWP carried out the experiments. ZL and CSC designed the study and drafted the manuscript. DYJ and KLS advised on experimental design. WJC provided cell lines and primary myeloma samples. All authors participated in the analysis and interpretation of data. All authors read and approved the final manuscript.

\section{Funding}

This work was supported by Hong Kong Blood Cancer Foundation and National Natural Science Foundation of China (81470369) awarded to CSC. The funders had no role in the design of the study and collection, analysis, and interpretation of data and in writing the manuscript.

\section{Availability of data and materials}

All data generated or analyzed during this study are included in this published article and its Additional files 1, 2,3.

\section{Ethics approval and consent to participate}

The study has been approved by Institutional Review Board of Queen Mary Hospital (UW 05-269T/932), and written informed consent has been obtained from patients for participation of this study.

\section{Consent for publication}

We have obtained consents from patients for publication of this report and any accompanying data or images.

\section{Competing interests}

The authors declare that they have no competing interests.

\section{Author details}

${ }^{1}$ Guangdong Key Laboratory of Genome Instability and Human Disease Prevention, Department of Biochemistry and Molecular Biology, Shenzhen University School of Medicine, Shenzhen, China. ${ }^{2}$ Department of Medicine, 
Queen Mary Hospital, The University of Hong Kong, Pokfulam Road, Pokfulam, Hong Kong, China. ${ }^{3}$ Division of Hematology, Mayo Clinic, Rochester, MN, USA. ${ }^{4}$ School of Biomedical Sciences, Queen Mary Hospital, The University of Hong Kong, Hong Kong, China. ${ }^{5}$ Department of Experimental Therapeutics, The University of Texas MD Anderson Cancer Center, Houston, TX, USA. ${ }^{6}$ Department of Haematology-Oncology, National University Cancer Institute, Singapore, Singapore.

Received: 15 January 2020 Accepted: 18 August 2020

Published online: 25 August 2020

\section{References}

1. Rajkumar SV. Multiple myeloma: 2016 update on diagnosis, risk-stratification, and management. Am J Hematol. 2016;91(7):719-34.

2. Rajkumar SV, Dimopoulos MA, Palumbo A, Blade J, Merlini G, Mateos MV, Kumar S, Hillengass J, Kastritis E, Richardson P, et al. International Myeloma Working Group updated criteria for the diagnosis of multiple myeloma. Lancet Oncol. 2014;15(12):e538-48.

3. Landgren $\mathrm{O}$, Kyle RA, Pfeiffer RM, Katzmann JA, Caporaso NE, Hayes RB, Dispenzieri A, Kumar S, Clark RJ, Baris D, et al. Monoclonal gammopathy of undetermined significance (MGUS) consistently precedes multiple myeloma: a prospective study. Blood. 2009;113(22):5412-7.

4. Morgan GJ, Walker BA, Davies FE. The genetic architecture of multiple myeloma. Nat Rev Cancer. 2012;12(5):335-48.

5. Chim CS, Kumar SK, Orlowski RZ, Cook G, Richardson PG, Gertz MA, Giralt S, Mateos MV, Leleu X, Anderson KC. Management of relapsed and refractory multiple myeloma: novel agents, antibodies, immunotherapies and beyond. Leukemia. 2018;32(2):252-62.

6. Bai Y, Orfao A, Chim CS. Molecular detection of minimal residual disease in multiple myeloma. Br J Haematol. 2018;181(1):11-26.

7. Mercer TR, Dinger ME, Mattick JS. Long non-coding RNAs: insights into functions. Nat Rev Genet. 2009;10(3):155-9.

8. Spizzo R, Almeida MI, Colombatti A, Calin GA. Long non-coding RNAs and cancer: a new frontier of translational research? Oncogene. 2012;31(43):4577-87.

9. Wang KC, Yang YW, Liu B, Sanyal A, Corces-Zimmerman R, Chen Y, Lajoie BR, Protacio A, Flynn RA, Gupta RA, et al. A long noncoding RNA maintains active chromatin to coordinate homeotic gene expression. Nature. 2011;472(7341):120-4.

10. Ponting CP, Oliver PL, Reik W. Evolution and functions of long noncoding RNAs. Cell. 2009;136(4):629-41.

11. Nobili L, Ronchetti D, Agnelli L, Taiana E, Vinci C, Neri A. Long non-coding RNAs in multiple myeloma. Genes (Basel). 2018;9(2):69.

12. Nobili L, Lionetti M, Neri A. Long non-coding RNAs in normal and malignant hematopoiesis. Oncotarget. 2016;7(31):50666-81.

13. Meng YB, He X, Huang YF, Wu QN, Zhou YC, Hao DJ. Long noncoding RNA CRNDE promotes multiple myeloma cell growth by suppressing miR-451. Oncol Res. 2017;25(7):1207-14.

14. Yang $N$, Chen J, Zhang H, Wang X, Yao H, Peng Y, Zhang W. LncRNA OIP5AS1 loss-induced microRNA-410 accumulation regulates cell proliferation and apoptosis by targeting KLF10 via activating PTEN/PI3K/AKT pathway in multiple myeloma. Cell Death Dis. 2017:8(8):e2975.

15. Shen H, Laird PW. Interplay between the cancer genome and epigenome. Cell. 2013;153(1):38-55.

16. Jones PA, Baylin SB. The fundamental role of epigenetic events in cancer. Nat Rev Genet. 2002;3(6):415-28.

17. Fazzari MJ, Greally JM. Epigenomics: beyond CpG islands. Nat Rev Genet. 2004;5(6):446-55.

18. Gardiner-Garden M, Frommer M. CpG islands in vertebrate genomes. J Mol Biol. 1987;196(2):261-82.

19. Antequera F. Structure, function and evolution of $\mathrm{CpG}$ island promoters. Cell Mol Life Sci. 2003;60(8):1647-58.

20. Robertson KD, Wolffe AP. DNA methylation in health and disease. Nat Rev Genet. 2000;1(1):11-9.

21. Guillamot M, Cimmino L, Aifantis I. The impact of DNA methylation in hematopoietic malignancies. Trends Cancer. 2016;2(2):70-83.

22. Chim CS, Liang R, Kwong YL. Hypermethylation of gene promoters in hematological neoplasia. Hematol Oncol. 2002;20(4):167-76.
23. Lopez-Serra P, Esteller M. DNA methylation-associated silencing of tumorsuppressor microRNAs in cancer. Oncogene. 2012;31(13):1609-22.

24. Wong KY, Chim CS. DNA methylation of tumor suppressor proteincoding and non-coding genes in multiple myeloma. Epigenomics. 2015;7(6):985-1001.

25. Chim CS, Kwong YL, Liang R. Gene hypermethylation in multiple myeloma: lessons from a cancer pathway approach. Clin Lymphoma Myeloma. 2008;8(6):331-9.

26. Wong KY, Li Z, Zhang X, Leung GK, Chan GC, Chim CS. Epigenetic silencing of a long non-coding RNA KIAA0495 in multiple myeloma. Mol Cancer. 2015;14:175.

27. Park SM, Park SJ, Kim HJ, Kwon OH, Kang TW, Sohn HA, Kim SK, Moo Noh S, Song KS, Jang SJ, et al. A known expressed sequence tag, BM742401, is a potent lincRNA inhibiting cancer metastasis. Exp Mol Med. 2013;45:e31.

28. Wang LQ, Wong KY, Li ZH, Chim CS. Epigenetic silencing of tumor suppressor long non-coding RNA BM742401 in chronic lymphocytic leukemia. Oncotarget. 2016;7(50):82400-10.

29. International Myeloma Working Group. Criteria for the classification of monoclonal gammopathies, multiple myeloma and related disorders: a report of the International Myeloma Working Group. Br J Haematol. 2003;121(5):749-57.

30. Greipp PR, San Miguel J, Durie BG, Crowley JJ, Barlogie B, Blade J, Boccadoro M, Child JA, Avet-Loiseau H, Kyle RA, et al. International staging system for multiple myeloma. J Clin Oncol. 2005;23(15):3412-20.

31. Kumar S, Paiva B, Anderson KC, Durie B, Landgren O, Moreau P, Munshi N, Lonial S, Blade J, Mateos MV, et al. International Myeloma Working Group consensus criteria for response and minimal residual disease assessment in multiple myeloma. Lancet Oncol. 2016;17(8):e328-46.

32. Wong KY, Wan TS, So CC, Chim CS. Establishment of a bortezomib-resistant Chinese human multiple myeloma cell line: MMLAL. Cancer Cell Int. 2013;13(1):122

33. Li Z, Wong KY, Chan GC, Chim CS. Epigenetic silencing of LPP/miR-28 in multiple myeloma. J Clin Pathol. 2018;71(3):253-8.

34. Li Z, Wong KY, Chan GC, Chng WJ, Chim CS. Epigenetic silencing of EVL/ miR-342 in multiple myeloma. Transl Res. 2018;192:46-53.

35. Yuan L, Chan GC, Fung KL, Chim CS. RANKL expression in myeloma cells is regulated by a network involving RANKL promoter methylation, DNMT1, microRNA and TNFalpha in the microenvironment. Biochim Biophys Acta. 2014;1843(9):1834-8.

36. Ghobrial IM. Myeloma as a model for the process of metastasis: implications for therapy. Blood. 2012;120(1):20-30.

37. Chim CS, Fung TK, Cheung WC, Liang R, Kwong YL. SOCS1 and SHP1 hypermethylation in multiple myeloma: implications for epigenetic activation of the Jak/STAT pathway. Blood. 2004;103(12):4630-5.

38. Chim CS, Pang R, Fung TK, Choi CL, Liang R. Epigenetic dysregulation of Wnt signaling pathway in multiple myeloma. Leukemia. 2007;21(12):2527-36.

39. Wong $K Y$, Yim RL, So CC, Jin DY, Liang R, Chim CS. Epigenetic inactivation of the MIR34B/C in multiple myeloma. Blood. 2011;118(22):5901-4.

40. Wong KY, Liang R, So CC, Jin DY, Costello JF, Chim CS. Epigenetic silencing of MIR203 in multiple myeloma. Br J Haematol. 2011;154(5):569-78.

41. Lujambio A, Esteller M. CpG island hypermethylation of tumor suppressor microRNAs in human cancer. Cell Cycle. 2007;6(12):1455-9.

42. Wang LQ, Kwong YL, Kho CS, Wong KF, Wong KY, Ferracin M, Calin GA, Chim CS. Epigenetic inactivation of miR-9 family microRNAs in chronic lymphocytic leukemia-implications on constitutive activation of NFkappaB pathway. Mol Cancer. 2013;12:173.

43. Wong KY, Yim RL, Kwong YL, Leung CY, Hui PK, Cheung F, Liang R, Jin DY, Chim CS. Epigenetic inactivation of the MIR129-2 in hematological malignancies. J Hematol Oncol. 2013;6:16.

44. Galm O, Wilop S, Reichelt J, Jost E, Gehbauer G, Herman JG, Osieka R. DNA methylation changes in multiple myeloma. Leukemia. 2004;18(10):1687-92.

45. Guillerm G, Depil S, Wolowiec D, Quesnel B. Different prognostic values of p15(INK4b) and p16(INK4a) gene methylations in multiple myeloma. Haematologica. 2003;88(4):476-8.

46. Chim CS. Updated survivals and prognostic factor analysis in myeloma treated by a staged approach use of bortezomib/thalidomide/dexamethasone in transplant eligible patients. J Transl Med. 2010;8:124.

47. Anderson KC, Carrasco RD. Pathogenesis of myeloma. Annu Rev Pathol. 2011:6:249-74. 
48. Weidenfeld J, Shu W, Zhang L, Millar SE, Morrisey EE. The WNT7b promoter is regulated by TTF-1, GATA6, and Foxa2 in lung epithelium. J Biol Chem. 2002;277(23):21061-70.

49. Ekroll IK, Avdal J, Swillens A, Torp H, Lovstakken L. An extended least squares method for aliasing-resistant vector velocity estimation. IEEE Trans Ultrason Ferroelectr Freq Control. 2016;63(11):1745-57.

\section{Publisher's Note}

Springer Nature remains neutral with regard to jurisdictional claims in published maps and institutional affiliations.
Ready to submit your research? Choose BMC and benefit from:

- fast, convenient online submission

- thorough peer review by experienced researchers in your field

- rapid publication on acceptance

- support for research data, including large and complex data types

- gold Open Access which fosters wider collaboration and increased citations

- maximum visibility for your research: over 100M website views per year

At BMC, research is always in progress.

Learn more biomedcentral.com/submissions 